\title{
Title: Preoperative albumin-to-fibrinogen ratio predicts postoperative pulmonary complications in patients after minimally invasive esophagectomy
}

\section{Zhang Peng}

The First Affiliated Hospital of Zhengzhou University

Zhao Song ( $\nabla$ zhaosong@zzu.edu.cn )

The First Affiliated Hospital of Zhengzhou University

\section{Research Article}

Keywords: nutrition, esophageal cancer, MIE, PPCs

Posted Date: June 15th, 2021

DOl: https://doi.org/10.21203/rs.3.rs-604731/v1

License: (c) (i) This work is licensed under a Creative Commons Attribution 4.0 International License.

Read Full License 


\section{Abstract \\ Background}

Postoperative pulmonary complications (PPCs) are the most common postoperative complications in patients with esophageal cancer. Prediction of PPCs by establishing a preoperative physiological function parameter model can help patients make adequate preoperative preparation, reduce treatment costs, and improve prognosis and quality of life. The purpose of this study was to investigate the relationship between albumin-to-fibrinogen ratio (AFR), prognostic nutritional index (PNI), albumin-toglobulin ratio (AGR), neutrophils-to-lymphocyte ratio (NLR), platelet-to-lymphocyte (PLR), and monocyteto -lymphocyte ratio (MLR) and other preoperative laboratory tests and PPCs in patients after esophagectomy.

\section{Methods}

Retrospective analysis was performed on total 712 consecutive patients who underwent esophagectomy in the Department of Thoracic Surgery, The First Affiliated Hospital of Zhengzhou University from July 2018 to December 2020. Patients were divided into training (535 patients) and validation (177) groups for comparison of baseline data, perioperative indicators, and laboratory examination data. Receiver operating characteristic (ROC) curve analysis was used to evaluate the efficacy, sensitivity and specificity of AFR, and Youden's index was used to calculate the cut-off values of AFR. Univariate and multivariate logistic regression analyses were used to assess the risk factors for PPCs in training group.

\section{Results}

$112(20.9 \%)$ in training group and $36(20.3 \%)$ in validation group developed PPCs. The AUC value predicted by AFR using ROC curve analysis was 0.817 , sensitivity $76.2 \%$ and specificity $78.7 \%$ in training group while AUC 0.803 , sensitivity $69.4 \%$ and specificity $85.8 \%$. Multivariate logistic regression analysis showed that smoking index, American Society of Anesthesiologists (ASA), AFR, and recurrent laryngeal nerve palsy were independent risk factors for PPCs.

\section{Conclusion}

Preoperative AFR can effectively predict the occurrence of PPCs in patients with esophageal cancer

\section{Background}

Esophageal cancer is a common malignant tumor worldwide, with a wide range of distribution and a high mortality rate, which brings a great burden of life to people around the world ${ }^{1}$. In a study on the causes of death of 508,585 cancer patients worldwide in 2018 , the incidence of esophageal cancer ranks the 
seventh in the world, and the mortality ranks the sixth ${ }^{2}$. At present, surgical resection for early esophageal cancer or neoadjuvant chemoradiotherapy followed by esophagectomy for locally advanced esophageal cancer $^{3}$ is still the main treatment method. Recently, minimally invasive esophagectomy (MIE) has gradually become the mainstream instead of traditional open surgery. A Randomized Controlled Trial (RCT) showed MIE reduced hospital stay, lung infection, and short-term quality of life compared with traditional open surgery ${ }^{4}$, meanwhile several studies have shown that the incidence of postoperative pulmonary complications (PPC) after esophagectomy is still between $20 \%$ and $40 \%$, which is the most common postoperative complication ${ }^{5}$, and postoperative pneumonia, as the most common pulmonary complication, seriously affects the prognosis and the quality of life of patients with esophageal cancer ${ }^{6}$. Therefore, the establishment of a model to predict the possibility of PPC is always required. Preoperative laboratory tests, such as white blood cell, albumin, globulin, are usually readily available for us. If we can find the association between laboratory tests and postoperative PPC would be able to help patients make adequate preoperative preparation, reduce treatment costs, improve prognosis and quality of life.

In 2002, T. Nozoel proposed that preoperative nutritional status might predict postoperative complications in patients with esophageal cancer ${ }^{7}$. Since then, a variety of nutritional factors, including neutrophils, lymphocytes, C-reactive protein, albumin, fibrinogen and related ratios, even grip strength, have been reported to be independent predictors of postoperative complications and prognosis ${ }^{8-11}$. Albumin, as an important biochemical index to evaluate nutritional status, has been shown to be associated with postoperative complications and prognosis in patients with a variety of tumors, including esophageal cancer ${ }^{11-15}$. Meanwhile, fibrinogen, as an indicator of blood coagulation, has a relatively short half-life and can be used as an acute response protein to reflect systemic inflammation. Guan.el proposed that low preoperative fibrinogen level may be a potential risk factor for neurological complications after cardiac surgery ${ }^{16}$. Then, a new indicator, albumin-to-fibrinogen ratio (AFR), was proposed in a study to predict postoperative severe complications in elderly patients with gastric cancer ${ }^{17}$. Although AFR has been proven to be associated with the prognosis of esophageal cancer ${ }^{18}$, it is still unknown whether AFR can predict the occurrence of PPC in patients with esophageal cancer. Therefore, this study was devoted to exploring the relationship between AFR and other well-known nutritional index and PPC after esophagectomy.

\section{Material And Methods}

\section{Patients}

A total of 712 consecutive patients who underwent radical MIE in the Department of Thoracic Surgery, the First Affiliated Hospital of Zhengzhou University from July 2018 to December 2020 were divided into two groups. The first group as the training group included 535 patients who underwent MIE between July 2018 and December 2019, and another as the validation group included 177 patients between January 2020 and December 2020 was collected prospectively. A total of 37 cases were excluded, among them 7 cases were excluded due to tumor invasion, 21 cases were excluded due to lack of data, 7 cases were 
excluded due to open surgery, and 2 cases were excluded due to simultaneous total gastrectomy. All patients underwent preoperative routine examination and evaluation, including complete blood count, biochemical examination of liver and kidney function, blood coagulation examination, pulmonary function test, b-ultrasound of liver and gallbladder urinary system, b-ultrasound of the heart, chest and upper abdomen enhanced Computed Tomography (CT), skull CT, bone scan, electrocardiogram, and gastroscopy. If the patient's general condition allowed, neoadjuvant chemoradiotherapy or chemotherapy was performed after discussion in the thoracic department. One day before the operation, department experts and anesthesiologists discussed and assessed the risks of the operation and preliminarily formulated the operation method. All the patients included in this study underwent three types of surgery including Mckeown, Ivor Lewis and Sweet, but not traditional open surgery. The recurrent laryngeal nerve and thoracic duct should be protected during mediastinal stage. The reconstruction of digestive tract was performed by replacing esophagus with tubular stomach, and the location of the anastomotic site was built in neck or chest depending on the patient's condition and tumor location. Finally, the anastomotic site was wrapped around with greater omentum or a kind of high molecular tissue material which has been proved to prevent anastomotic leakage in our center ${ }^{19}$. Postoperative pathology showed that all the 712 patients had negative resection margin, which means R0 resection. The first day after the operation, bedside chest radiograph was usually performed. Complete blood count, electrolyte examination, liver and kidney function tests were performed on the 1st and 3rd day after the operation. Chest CT was scheduled on the third day after surgery. Tracheal sputum was collected from all patients with suspected pulmonary infection for culture, and the treatment regimen was adjusted on day 2 or 3 according to the patient's clinical manifestations and sputum culture results. All patients received oxygen inhalation, sputum - reducing drug inhalation, injection or analgesics, and intravenous infusion within a few days after surgery. When phlegm is sticky and difficult to cough out, affecting respiration, the patient will be made bedside or tracheobronchial mirror room sputum suction treatment. Anyone who may have developed respiratory failure or other new organ dysfuntion is transferred to the intensive care unit (ICU) for treatment as soon as possible. The retrospective study was approved by the Ethics Committee of the First Affiliated Hospital of Zhengzhou University. In addition to this, we confirm that all methods were performed in accordance with the relevant guidelines and regulations. Written informed consent was obtained from all eligible patients.

\section{Definition of postoperative pulmonary complications}

The occurrence of PPCs was determined if any of the following occurred in hospital within 7 days: ${ }^{20}$ atelectasis, pleural effusion, pneumothorax, bronchospasm, respiratory infection, respiratory failure, or aspiration pneumonitis. Atelectasis, pleural effusion and pneumothorax were diagnosed by X-rays or CT. Bronchospasm was defined as newly detected expiratory wheezing that required treatment with bronchodilators. The revised Uniform Pneumonia Score (rUPS) was used to define postoperative pneumonia. The score was based on the following three factors: body temperature $\left({ }^{\circ} \mathrm{C}\right)(\geq 36.1$ and $\leq$ $38.4=0, \leq 36.0$ or $\geq 38.5=1)$ and white blood cell count $\left(\times 10^{9} / L\right)(\geq 4.0$ and $\leq 11.0=0,<4.0$ or $>11.0=$ 1) and pulmonary radiological results (chest $X$-ray and/or CT scan, CT preferred) (no infiltrate $=0$, diffused(or patchy) infiltrate $=1$, well-circumscribed infiltrate $=2$ ). A sum score of 2 points or higher, of 
which at least 1 point is assigned due to infiltrative findings on pulmonary radiography, indicates treatment of pneumonia ${ }^{21}$. Respiratory failure is defined as partial arterial oxygen pressure of $60 \mathrm{mmHg}$ in indoor air, partial arterial oxygen pressure/graded inhalation oxygen concentration of 300 , or oxygen therapy is required and arterial oxygen saturation is $90 \% 22$.

\section{Data collection}

Preoperative laboratory tests of all patients were completed within one week before surgery. Baseline data included sex, age, comorbidities, and perioperative indicators. The severity of smoking was indicated by the smoking index (cigarettes/day $\times$ years). In addition, based on previous studies, we calculated the following indicators: prognostic nutritional index $(\mathrm{PNI})$ : $10 \times$ serum albumin $(\mathrm{g} / \mathrm{dL}) \otimes 0.005 \times$ total lymphocyte count (per $\left.\mathrm{mm}^{3}\right)^{23}$, AFR as mentioned, albumin-to-globulin ratio (AGR), neutrophils-tolymphocyte ratio (NLR), platelet-to-lymphocyte (PLR), and monocyte-to -lymphocyte ratio (MLR).

\section{Statistical analysis}

Receiver operating characteristic (ROC) curve analysis was established for determining the predictive value and cut-off value of AFR for PPCs. Training group was divided into two groups with and without PPCs, and the validation group was divided into high and low AFR according to cut-off value of AFR obtained by the training group. Meanwihle, based on the cut-off value in the training group, high and low AFR were used for ROC curve analysis both in training goup and validation group. All variables are expressed as means \pm standard deviation (SD) or numbers with percentage $(n, \%)$. Continuous variables were compared using Mann-Whitney $U$ test or Student $t$ test, whereas categorical variables using Chisquare test or Fisher exact test as appropriate, and training data with statistically significant differences was included in univariate and multivariate logistic analysis. All the probabilities are double-tailed probabilities. When $P$ value is less than 0.05 , there is a statistical difference. All statistical analyses were performed by SPSS version 22 (IBM Corporation, Armonk, NY, USA).

\section{Result}

\section{Baseline data of patients}

Baseline information and perioperative data of training group was listed in Table 1. The average age was 65, with 144 females (26.9\%) and 391 males (73.1\%). According to whether the patients had PPCs, they were divided into the PPC group and the non-PPC group, of which 112 (20.9\%) were in the PPC group. The results showed that age, history of smoking, smoking index, FEV1/pre, PNI, AFR, AGR, the American Society of Anesthesiologists (ASA) physical status, recurrent laryngeal nerve palsy, days in ICU and days in hospital after surgery were all significantly different between the two groups $(p<0.05)$. Others were not statistically significant $(p>0.05)$. Then, as Table 2 showed, all possible risk factors $(p<0.05)$ were included in univariate logistic regression analysis. Variables with $p<0.1$ in univariate analysis were included in multivariate analysis. Multivariate logistic regression analysis revealed that smoking index (odds ratio [OR] 1.001, 95\% confidence interval [CI] 1.000-1.002, $\mathrm{p}=0.024)$, AFR (OR 0.599, 95\% Cl 0.511- 
$0.702, \mathrm{p}<0.001)$, ASA physical status $\otimes(\mathrm{OR} 7.865,95 \% \mathrm{Cl} 1.684-36.737, \mathrm{p}=0.009)$ and recurrent laryngeal nerve palsy (OR $3.721,95 \% \mathrm{Cl} 1.044-13.263, \mathrm{p}=0.043)$ were independent risk factors of PPC. 
Table 1

Demographic and clinical characteristics in training group

\begin{tabular}{|c|c|c|c|c|}
\hline Variables & $\begin{array}{l}\text { All patients [n } \\
=535]\end{array}$ & $\begin{array}{l}\text { PPC group [n } \\
=122]\end{array}$ & $\begin{array}{l}\text { Non-PPC group [n } \\
=413]\end{array}$ & $\begin{array}{l}p- \\
\text { Value }\end{array}$ \\
\hline Gender, n (\%) & & & & 0.756 \\
\hline Male & $391(73.1)$ & $91(74.6)$ & $300(72.6)$ & - \\
\hline Female & $144(26.9)$ & $31(25.4)$ & $113(27.4)$ & - \\
\hline Age, years $($ mean $\pm S D)$ & $64.5 \pm 7.7$ & $65.9 \pm 6.3$ & $64.1 \pm 8.0$ & $0.011^{*}$ \\
\hline $\mathrm{BMI}, \mathrm{kg} / \mathrm{m}^{2}($ mean $\pm \mathrm{SD})$ & $23.7 \pm 3.2$ & $23.3 \pm 3.5$ & $23.8 \pm 3.1$ & 0.134 \\
\hline \multicolumn{5}{|l|}{ Comorbidities, n (\%) } \\
\hline Hypertension & $185(34.6)$ & $41(33.6)$ & $144(34.9)$ & 0.797 \\
\hline Cerebral infarction & $59(11.0)$ & $17(13.9)$ & $42(10.2)$ & 0.243 \\
\hline Coronary heart disease & $70(13.1)$ & $13(10.7)$ & $57(13.8)$ & 0.365 \\
\hline COPD & $39(7.3)$ & $13(10.7)$ & $26(6.3)$ & 0.104 \\
\hline Hepatitis & $17(3.2)$ & $5(4.1)$ & $12(2.9)$ & 0.509 \\
\hline Diabetes & $67(12.5)$ & $15(12.3)$ & $52(12.6)$ & 1.000 \\
\hline History of smoking, $\mathrm{n}(\%)$ & & & & $<$ \\
\hline Non-smoker & & & & $0.001^{*}$ \\
\hline Ex-smoker & $322(60.2)$ & $56(45.9)$ & $266(64.4)$ & - \\
\hline \multirow[t]{2}{*}{ Current-smoker } & $76(14.2)$ & 17 (13.9) & $59(14.3)$ & - \\
\hline & $137(15.6)$ & $49(40.2)$ & $88(21.3)$ & - \\
\hline Smoking index (mean $\pm S D)$ & $279.3 \pm 499.0$ & $481.2 \pm 698.9$ & $219.7 \pm 404.5$ & $\begin{array}{l}<.001^{*} \\
\end{array}$ \\
\hline History of drinking, $\mathrm{n}$ (\%) & & & & 0.226 \\
\hline Yes & $148(17.7)$ & $39(32.0)$ & $109(26.4)$ & - \\
\hline No & $387(72.3)$ & $83(68.0)$ & $304(73.6)$ & - \\
\hline
\end{tabular}

Note: Continuous variables are presented as mean \pm standard deviation, and categorical variables are presented as number (\%). $p$-Value were calculated by Chi-square test, Fisher exact test, MannWhitney U or T test.

Abbreviations: $P P C$ postoperative pulmonary complication, $B M /$ body mass index, $C O P D$ chronic obstructive pulmonary disease, $F V C /$ pre forced votal capacity/predicion, $F E V 1 /$ pre forced expiratory volumn in one second/prediction, $P N /$ prognostic nutritional index, AFR albumin-to-fibrinogen ratio, $A G R$ albumin-to-globin ratio, NLR neutrophils-to-lymphocyte ratio, $P L R$ platelet-to-lymphocyte ratio, $M L R$ monocytes-lymphocyte ratio, ASA American Society of Anesthesiologists, ICU intensive care unit 


\begin{tabular}{|c|c|c|c|c|}
\hline Variables & $\begin{array}{l}\text { All patients [n } \\
=535]\end{array}$ & $\begin{array}{l}\text { PPC group [n } \\
=122]\end{array}$ & $\begin{array}{l}\text { Non-PPC group [n } \\
=413]\end{array}$ & $\begin{array}{l}p- \\
\text { Value }\end{array}$ \\
\hline History of chest surgery, n (\%) & $9(1.7)$ & $3(2.5)$ & $6(1.5)$ & 0.433 \\
\hline Location,n (\%) & & & & 0.382 \\
\hline Upper & $42(7.9)$ & $11(9.0)$ & $31(7.5)$ & - \\
\hline Middle & $308(57.6)$ & $71(58.2)$ & $237(57.4)$ & - \\
\hline Lower & 168 (31.4) & $39(32.0)$ & $129(31.2)$ & - \\
\hline Esophagogastric junction & $17(3.2)$ & $1(0.8)$ & $16(3.9)$ & - \\
\hline Tumor length, $\mathrm{cm}($ mean $\pm \mathrm{SD})$ & $3.1 \pm 1.6$ & $3.0 \pm 1.6$ & $3.2 \pm 1.6$ & 0.414 \\
\hline FVC/pre, \% (mean \pm SD) & $104.9 \pm 16.1$ & $105.5 \pm 17.0$ & $104.7 \pm 15.9$ & 0.624 \\
\hline FEV1/pre, \% (mean \pm SD) & $76.6 \pm 8.7$ & $74.9 \pm 6.2$ & $77.2 \pm 9.2$ & $0.002^{*}$ \\
\hline \multicolumn{5}{|l|}{$\begin{array}{l}\text { Preoperative laboratory tests } \\
\text { (mean } \pm \text { SD) }\end{array}$} \\
\hline \multirow{2}{*}{ White blood cells, mm } & $6.0 \pm 2.0$ & $6.1 \pm 1.7$ & $5.9 \pm 2.0$ & 0.625 \\
\hline & $129.1 \pm 15.3$ & $128.7 \pm 15.4$ & $129.2 \pm 15.3$ & 0.781 \\
\hline Creatinine, $\mathrm{mg} / \mathrm{dL}$ & $67.4 \pm 17.8$ & $71.2 \pm 21.0$ & $66.2 \pm 16.6$ & 0.265 \\
\hline $\mathrm{PNI}$ & $40.2 \pm 5.0$ & $37.1 \pm 4.7$ & $41.1 \pm 4.7$ & $\begin{array}{l}< \\
0.001^{*}\end{array}$ \\
\hline $\begin{array}{l}\text { AFR } \\
\text { AGR }\end{array}$ & $12.4 \pm 2.7$ & $10.2 \pm 2.4$ & $13.0 \pm 2.5$ & $\begin{array}{l}< \\
0.001^{*}\end{array}$ \\
\hline $\begin{array}{l}\text { NLR } \\
\text { PLR }\end{array}$ & $1.6 \pm 0.4$ & $1.4 \pm 0.3$ & $1.7 \pm 0.4$ & $\begin{array}{l}< \\
0.001^{*}\end{array}$ \\
\hline \multirow[t]{3}{*}{ MLR } & $2.8 \pm 4.3$ & $3.3 \pm 4.8$ & $2.6 \pm 4.1$ & 0.136 \\
\hline & $90.3 \pm 50.0$ & $88.5 \pm 43.5$ & $90.8 \pm 51.7$ & 0.645 \\
\hline & $0.3 \pm 0.2$ & $0.3 \pm 0.2$ & $0.3 \pm 0.1$ & 0.486 \\
\hline Neoadjuvant therapy, n (\%) & & & & 0.271 \\
\hline
\end{tabular}

Yes

Note: Continuous variables are presented as mean \pm standard deviation, and categorical variables are presented as number (\%). $p$-Value were calculated by Chi-square test, Fisher exact test, MannWhitney U or T test.

Abbreviations: $P P C$ postoperative pulmonary complication, $B M /$ body mass index, $C O P D$ chronic obstructive pulmonary disease, $F V C /$ pre forced votal capacity/predicion, $F E V 1 /$ pre forced expiratory volumn in one second/prediction, $P N /$ prognostic nutritional index, $A F R$ albumin-to-fibrinogen ratio, $A G R$ albumin-to-globin ratio, NLR neutrophils-to-lymphocyte ratio, $P L R$ platelet-to-lymphocyte ratio, $M L R$ monocytes-lymphocyte ratio, ASA American Society of Anesthesiologists, ICU intensive care unit 


\begin{tabular}{llll}
$\begin{array}{l}\text { All patients [n } \\
=535]\end{array}$ & $\begin{array}{l}\text { PPC group [n } \\
=122\end{array}$ & $\begin{array}{l}\text { Non-PPC group [n } \\
=413]\end{array}$ & $\begin{array}{l}p- \\
\text { Value }\end{array}$ \\
\hline $150(28.0)$ & $39(32.0)$ & $111(26.9)$ & - \\
$385(72.0)$ & $83(68.0)$ & $302(73.1)$ & -
\end{tabular}

ASA Physical Status, $\mathrm{n}(\%)$

\begin{tabular}{|lll|}
\hline $44(8.2)$ & $2(1.7)$ & $42(10.2)$ \\
\hline $416(77.8)$ & $105(87.5)$ & $311(75.7)$ \\
\hline $71(13.3)$ & $13(10.8)$ & $58(14.1)$
\end{tabular}

Type of esophagectomy, n (\%)

Mckeown

507 (94.8)

118 (96.7)

389 (94.2)

Ivor Lewis

$6(1.1)$

$1(0.8)$

$5(1.2)$

Sweet

22 (4.1)

$3(2.5)$

$19(4.6)$

Length of operation, min (mean

\pm SD)

$335.7 \pm 73.5$

$335.7 \pm 75.5$

$335.7 \pm 72.9$

1.000

Blood loss, $\mathrm{ml}$ (mean \pm SD)

$163.5 \pm 78.1$

$159.6 \pm 73.4$

$164.6 \pm 79.5$

0.537

Site of anastomotic, $\mathrm{n}(\%)$

Neck

Chest

Histology, n (\%)

Squamous cell carcinoma

Adenocarcinoma

Small cell carcinoma

Others

Pathological depth of invasion, $\mathrm{n}$

(\%)

PTO

\begin{tabular}{lll}
\hline $504(94.2)$ & $116(95.1)$ & $388(94.2)$ \\
\hline $30(5.6)$ & $6(4.9)$ & $24(5.8)$
\end{tabular}

0.702

Nete: Continuous variables are presented as mean \pm standard deviation, and categorical variables are presented as number (\%). $p$-Value were calculated by Chi-square test, Fisher exact test, MannWHhitney U or T test.

Apbreviations: $P P C$ postoperative pulmonary complication, $B M /$ body mass index, $C O P D$ chronic obstructive pulmonary disease, $F V C /$ pre forced votal capacity/predicion, FEV1/pre forced expiratory pqlyimn in one second/prediction, $P N /$ prognostic nutritional index, $A F R$ albumin-to-fibrinogen ratio, $A G R$ albumin-to-globin ratio, NLR neutrophils-to-lymphocyte ratio, $P L R$ platelet-to-lymphocyte ratio, $M L R$ monocytes-lymphocyte ratio, ASA American Society of Anesthesiologists, ICU intensive care unit 


\begin{tabular}{|c|c|c|c|c|}
\hline Variables & $\begin{array}{l}\text { All patients [n } \\
=535]\end{array}$ & $\begin{array}{l}\text { PPC group [n } \\
=122]\end{array}$ & $\begin{array}{l}\text { Non-PPC group [n } \\
=413]\end{array}$ & $\begin{array}{l}p- \\
\text { Value }\end{array}$ \\
\hline \multirow{5}{*}{$\begin{array}{l}\text { Pathological depth of invasion, } n \\
(\%)\end{array}$} & $105(19.6)$ & $25(20.5)$ & $80(19.4)$ & - \\
\hline & $125(23.4)$ & $25(20.5)$ & $100(24.2)$ & - \\
\hline & $159(29.7)$ & $37(30.3)$ & $122(29.5)$ & - \\
\hline & $75(14.0)$ & $16(13.1)$ & $59(14.3)$ & - \\
\hline & & & & 0.560 \\
\hline \multirow{2}{*}{ pN0 } & $347(64.9)$ & $78(63.9)$ & $269(65.1)$ & - \\
\hline & $110(20.6)$ & $23(18.9)$ & $87(21.1)$ & - \\
\hline \multirow{2}{*}{$\begin{array}{l}\text { pN2 } \\
\text { pN3 }\end{array}$} & $61(11.4)$ & $18(14.8)$ & $43(10.4)$ & - \\
\hline & $17(3.2)$ & $14(3.4)$ & $3(2.5)$ & - \\
\hline $\begin{array}{l}\text { Pathological distant metastasis, } \\
\text { n (\%) }\end{array}$ & & & & 0.132 \\
\hline pM0 & $532(99.4)$ & $120(98.4)$ & $412(99.8)$ & - \\
\hline pM1 & $3(0.6)$ & $2(1.6)$ & $1(0.2)$ & - \\
\hline \multicolumn{2}{|l|}{ Histologic grade, n (\%) } & & & 0.104 \\
\hline G1 & $77(14.4)$ & $18(14.8)$ & $59(14.3)$ & - \\
\hline $\mathrm{G} 1-2$ & $39(7.3)$ & $9(7.4)$ & $30(7.3)$ & - \\
\hline $\mathrm{G} 2$ & $313(58.5)$ & $63(51.6)$ & $250(60.5)$ & - \\
\hline $\mathrm{G} 2-3$ & $57(10.7)$ & $21(17.2)$ & $36(8.7)$ & - \\
\hline G3 & $49(9.2)$ & $11(9.0)$ & $38(9.2)$ & - \\
\hline \multicolumn{5}{|l|}{$\begin{array}{l}\text { Postoperative complications, } n \\
\text { (\%) }\end{array}$} \\
\hline \multirow{2}{*}{$\begin{array}{l}\text { Anastomotic leak } \\
\text { Recurrent laryngeal nerve palsy }\end{array}$} & $42(7.9)$ & $5(4.1)$ & $37(9.0)$ & 0.079 \\
\hline & $17(3.2)$ & $8(6.6)$ & $9(2.2)$ & $0.033^{*}$ \\
\hline
\end{tabular}

Thoracic duct injury

Note Gontinuous variables are presented as mean \pm standard deviation, and categorical variables are presented as RUmber (\%). $p$-Value were calculated by Chi-square test, Fisher exact test, MannWhitney 4 or T test. Woundinfection

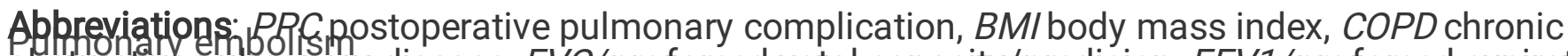
obstructive pulmonary disease, FVC/pre forced votal capacity/predicion, FEV1/pre forced expiratory kolump inone second/prediction, $P N /$ prognostic nutritional index, $A F R$ albumin-to-fibrinogen ratio, AGR a bumin-to-globin ratio, NLR neutrophils-to-lymphocyte ratio, $P L R$ platelet-to-lymphocyte ratio, MbRamenocytes-lymphocyte ratio, ASA American Society of Anesthesiologists, ICU intensive care unit 


\begin{tabular}{|c|c|c|c|c|}
\hline Variables & $\begin{array}{l}\text { All patients [n } \\
=535]\end{array}$ & $\begin{array}{l}\text { PPC group [n } \\
=122]\end{array}$ & $\begin{array}{l}\text { Non-PPC group [n } \\
=413]\end{array}$ & $p-$ \\
\hline & $6(1.1)$ & $0(0)$ & $6(1.5)$ & 0.345 \\
\hline & $16(3.0)$ & $1(0.8)$ & $15(3.6)$ & 0.137 \\
\hline & $2(0.4)$ & 0 & $2(0.5)$ & 1.000 \\
\hline & $3(0.6)$ & 0 & $3(0.7)$ & 1.000 \\
\hline & $1(0.2)$ & 0 & $1(0.2)$ & 1.000 \\
\hline & $3(0.6)$ & 0 & $3(0.7)$ & 1.000 \\
\hline $\begin{array}{l}\text { Days in ICU after surgery (mean } \\
\pm \text { SD) }\end{array}$ & $0.2 \pm 1.6$ & $0.6 \pm 2.8$ & $0.1 \pm 0.9$ & $0.005^{\star}$ \\
\hline $\begin{array}{l}\text { Days in hospital after surgery } \\
\text { (mean } \pm \text { SD) }\end{array}$ & $18.6 \pm 8.0$ & $20.6 \pm 8.1$ & $18.0 \pm 8.0$ & $<.001^{*}$ \\
\hline \multicolumn{5}{|c|}{$\begin{array}{l}\text { Note: Continuous variables are presented as mean } \pm \text { standard deviation, and categorical variables are } \\
\text { presented as number }(\%) . p \text {-Value were calculated by Chi-square test, Fisher exact test, Mann- } \\
\text { Whitney U or T test. }\end{array}$} \\
\hline \multicolumn{5}{|c|}{$\begin{array}{l}\text { Abbreviations: } P P C \text { postoperative pulmonary complication, } B M / \text { body mass index, } C O P D \text { chronic } \\
\text { obstructive pulmonary disease, } F V C / \text { pre forced votal capacity/predicion, } F E V 1 / p r e \text { forced expiratory } \\
\text { volumn in one second/prediction, } P N / \text { prognostic nutritional index, } A F R \text { albumin-to-fibrinogen ratio, } \\
A G R \text { albumin-to-globin ratio, } N L R \text { neutrophils-to-lymphocyte ratio, } P L R \text { platelet-to-lymphocyte ratio, } \\
M L R \text { monocytes-lymphocyte ratio, } A S A \text { American Society of Anesthesiologists, ICU intensive care unit }\end{array}$} \\
\hline
\end{tabular}


Table 2

Risk factors of PPC by univariate and multivariate logistic regression analyses

\begin{tabular}{|c|c|c|c|c|}
\hline \multirow[t]{2}{*}{ Variables } & \multicolumn{2}{|l|}{ Univariate analysis } & \multicolumn{2}{|l|}{ Multivariate analysis } \\
\hline & OR $(95 \% \mathrm{Cl})$ & $\begin{array}{l}p- \\
\text { Value }\end{array}$ & OR $(95 \% \mathrm{Cl})$ & $\begin{array}{l}p- \\
\text { Value }\end{array}$ \\
\hline \multicolumn{5}{|l|}{ Gender } \\
\hline female (referrence) & $0.904(0.570-1.435)$ & 0.670 & & \\
\hline Age & $1.032(1.004-1.060)$ & $0.024^{*}$ & $1.009(0.973-1.046)$ & 0.642 \\
\hline BMI & $0.951(0.891-1.016)$ & 0.135 & & \\
\hline \multicolumn{5}{|l|}{ COPD } \\
\hline no (referrence) & $1.775(0.883-3.571)$ & 0.107 & & \\
\hline \multicolumn{5}{|l|}{ History of smoking } \\
\hline Non-smoker (referrence) & 1.000 & & 1.000 & \\
\hline Current-smoker & $2.645(1.682-4.160)$ & $<0.001^{*}$ & $1.095(0.481-2.491)$ & 0.830 \\
\hline Ex-smoker & $1.369(0.742-2.523)$ & 0.315 & & \\
\hline Smoking index & $1.001(1.001-1.001)$ & $<0.001^{*}$ & $1.001(1.000-1.002)$ & $0.024^{\star}$ \\
\hline FEV1/pre & $0.971(0.949-0.993)$ & $0.011^{*}$ & $0.976(0.947-1.006)$ & 0.111 \\
\hline PNI & $0.842(0.804-0.881)$ & $<0.001^{*}$ & $0.976(0.912-1.045)$ & 0.492 \\
\hline AFR & $0.559(0.492-0.635)$ & $<0.001^{*}$ & $0.599(0.511-0.702)$ & $<0.001^{*}$ \\
\hline AGR & $0.175(0.091-0.335)$ & $<0.001^{*}$ & $0.652(0.296-1.436)$ & 0.288 \\
\hline \multicolumn{5}{|l|}{ Neoadjuvant therapy } \\
\hline no (referrence) & $1.278(0.825-1.982)$ & 0.272 & & \\
\hline \multicolumn{5}{|l|}{ ASA Physical Status } \\
\hline$\nabla($ referrence $)$ & 1.000 & & & \\
\hline प & $\begin{array}{l}7.090(1.687- \\
29.795)\end{array}$ & $0.007^{*}$ & $\begin{array}{l}7.865(1.684- \\
36.737)\end{array}$ & $0.009^{*}$ \\
\hline प & $\begin{array}{l}4.707(1.008- \\
21.972)\end{array}$ & $0.049^{*}$ & $\begin{array}{l}2.804(0.513- \\
15.318)\end{array}$ & 0.234 \\
\hline $\begin{array}{l}\text { Abbreviations: } P P C \text { posto } \\
\text { obstructive pulmonary di } \\
\text { prognostic nutritional ind } \\
\text { American Society of Ane }\end{array}$ & $\begin{array}{l}\text { pulmonary complicat } \\
\text { EV1/pre forced expira } \\
\text { albumin-to-fibrinogen } \\
\text { ggists, ICU intensive ca }\end{array}$ & $\begin{array}{l}\text { n, } B M / \mathrm{b} \\
\text { ry volum } \\
\text { atio, } A G R \\
\text { e unit, } O F\end{array}$ & $\begin{array}{l}\text { mass index, COPD chr } \\
\text { n one second/predictio } \\
\text { oumin-to-globin ratio, } A \\
\text { dds ratio, } C / \text { Confidence }\end{array}$ & $\begin{array}{l}\text { nic } \\
P N I \\
A \\
\text { interval }\end{array}$ \\
\hline
\end{tabular}




\begin{tabular}{|c|c|c|c|c|}
\hline \multirow[t]{2}{*}{ Variables } & \multicolumn{2}{|l|}{ Univariate analysis } & \multicolumn{2}{|l|}{ Multivariate analysis } \\
\hline & OR $(95 \% \mathrm{Cl})$ & $\begin{array}{l}p- \\
\text { Value }\end{array}$ & OR $(95 \% \mathrm{Cl})$ & $\begin{array}{l}p- \\
\text { Value }\end{array}$ \\
\hline \multicolumn{5}{|l|}{ Type of esophagectomy } \\
\hline Mckeown (referrence) & \multicolumn{4}{|l|}{1.000} \\
\hline Ivor Lewis & $0.659(0.076-5.699)$ & \multicolumn{3}{|l|}{0.705} \\
\hline Sweet & $0.521(0.151-1.790)$ & \multicolumn{3}{|l|}{0.521} \\
\hline \multicolumn{5}{|l|}{$\begin{array}{l}\text { Recurrent laryngeal nerve } \\
\text { palsy }\end{array}$} \\
\hline no (referrence) & $3.150(1.188-8.350)$ & $0.021^{*}$ & $\begin{array}{l}3.721(1.044- \\
13.263)\end{array}$ & $0.043^{*}$ \\
\hline Days in ICU after surgery & $1.178(1.024-1.356)$ & $0.022^{*}$ & $1.238(0.998-1.534)$ & 0.052 \\
\hline Days in hospital after surgery & $1.035(1.012-1.060)$ & $0.003^{*}$ & $1.021(0.990-1.052)$ & 0.182 \\
\hline \multicolumn{5}{|c|}{$\begin{array}{l}\text { Abbreviations: } P P C \text { postoperative pulmonary complication, } B M I \text { body mass index, } C O P D \text { chronic } \\
\text { obstructive pulmonary disease, } F E V 1 / p r e \text { forced expiratory volumn in one second/prediction, } P N I \\
\text { prognostic nutritional index, } A F R \text { albumin-to-fibrinogen ratio, } A G R \text { albumin-to-globin ratio, } A S A \\
\text { American Society of Anesthesiologists, ICU intensive care unit, } O R \text { Odds ratio, C/Confidence interval }\end{array}$} \\
\hline
\end{tabular}

Demographic and clinical characteristics in validation group were demonstrated in Table 3. The average age was 64, with 46 (26\%) females and 131 (74\%) males. Compared with the training group, only Mckeown and Ivor Lewis were operated, small cell carcinoma was not reported and no pulmonary embolism or mortality occurred. Between the high and low AFR group, gender, age, COPD, smoking index, FEV1/pre, ASA physical status, PPC and recurrent laryngeal nerve palsy were significantly different $(p<$ 0.05). 
Table 3

Demographic and clinical characteristics in validation group

\begin{tabular}{|c|c|c|c|c|}
\hline Variables & $\begin{array}{l}\text { All patients [n = } \\
\text { 177] }\end{array}$ & $\begin{array}{l}\mathrm{AFR} \geq 11.1[n= \\
129]\end{array}$ & $\begin{array}{l}\mathrm{AFR}<11.1[\mathrm{n}= \\
48]\end{array}$ & $p-$ \\
\hline Gender, n (\%) & & & & $0.013^{*}$ \\
\hline Male & $131(74.0)$ & $89(69.0)$ & $42(87.5)$ & - \\
\hline Female & $46(26.0)$ & $40(31.0)$ & $6(12.5)$ & - \\
\hline Age, years $($ mean $\pm S D)$ & $64.0 \pm 8.1$ & $63.1 \pm 8.1$ & $66.4 \pm 7.7$ & $0.017^{*}$ \\
\hline $\mathrm{BMI}, \mathrm{kg} / \mathrm{m}^{2}($ mean $\pm \mathrm{SD})$ & $23.9 \pm 3.0$ & $23.9 \pm 2.9$ & $23.9 \pm 3.5$ & 0.996 \\
\hline \multicolumn{5}{|l|}{ Comorbidities, n (\%) } \\
\hline Hypertension & $62(35.0)$ & $41(31.8)$ & $21(43.8)$ & 0.138 \\
\hline Cerebral infarction & $18(10.2)$ & $11(8.5)$ & $7(14.6)$ & 0.266 \\
\hline Coronary heart disease & $22(12.4)$ & $17(13.2)$ & $5(10.4)$ & 0.621 \\
\hline COPD & $9(5.1)$ & $3(2.3)$ & $6(12.5)$ & $0.013^{*}$ \\
\hline Hepatitis & $6(3.4)$ & $4(3.1)$ & $2(4.2)$ & 0.663 \\
\hline Diabetes & $22(12.4)$ & $13(10.1)$ & $9(18.8)$ & 0.120 \\
\hline History of smoking, n (\%) & & & & 0.073 \\
\hline Non-smoker & $91(51.4)$ & $73(56.6)$ & $18(37.5)$ & - \\
\hline Ex-smoker & $19(10.7)$ & $13(10.1)$ & $6(12.5)$ & - \\
\hline Current-smoker & $67(37.9)$ & 43 (33.3) & $24(50.0)$ & - \\
\hline Smoking index (mean $\pm S D$ ) & $389.7 \pm 635.0$ & $673.3 \pm 918.5$ & $219.7 \pm 404.5$ & $0.007^{*}$ \\
\hline History of drinking, n (\%) & & & & 0.767 \\
\hline Yes & $56(31.6)$ & $40(31.0)$ & $16(33.3)$ & - \\
\hline No & $121(68.4)$ & $89(69.0)$ & $32(66.7)$ & - \\
\hline History of chest surgery, $\mathrm{n}(\%)$ & $6(3.4)$ & $3(2.3)$ & $3(6.3)$ & 0.346 \\
\hline
\end{tabular}

Note: Continuous variables are presented as mean \pm standard deviation, and categorical variables are presented as number (\%). $p$-Value were calculated by Chi-square test, Fisher exact test, MannWhitney U or T test.

Abbreviations: $A F R$ albumin-to-fibrinogen ratio, $B M /$ body mass index, $C O P D$ chronic obstructive pulmonary disease, $F V C /$ pre forced votal capacity/predicion, $F E V 1 / p r e$ forced expiratory volumn in one second/prediction, ASA American Society of Anesthesiologists, PPC postoperative pulmonary complication, ICU intensive care unit 


\begin{tabular}{|c|c|c|c|c|}
\hline Variables & $\begin{array}{l}\text { All patients [n = } \\
177]\end{array}$ & $\begin{array}{l}\text { AFR } \geq 11.1[n= \\
129]\end{array}$ & $\begin{array}{l}\text { AFR }<11.1[n= \\
48]\end{array}$ & $\begin{array}{l}p- \\
\text { Value }\end{array}$ \\
\hline Location,n (\%) & & & & 0.352 \\
\hline Upper & $13(7.3)$ & $12(9.3)$ & $1(2.1)$ & - \\
\hline Middle & $84(47.5)$ & $62(48.1)$ & $22(45.8)$ & - \\
\hline Lower & $66(37.3)$ & $45(34.9)$ & $21(43.8)$ & - \\
\hline Esophagogastric junction & $14(7.9)$ & $1(0.8)$ & $16(3.9)$ & - \\
\hline Tumor length, n (\%) & & & & 0.326 \\
\hline$\geq 3$ & $100(56.5)$ & $70(54.3)$ & $30(62.5)$ & \\
\hline$<3$ & $77(43.5)$ & $59(45.7)$ & $18(37.5)$ & \\
\hline FVC/pre, \% (mean \pm SD) & $106.1 \pm 17.0$ & $107.3 \pm 17.4$ & $102.7 \pm 15.5$ & 0.110 \\
\hline FEV1/pre, \% (mean \pm SD) & $76.3 \pm 8.3$ & $75.2 \pm 7.6$ & $79.0 \pm 9.5$ & $0.007^{*}$ \\
\hline Neoadjuvant therapy, n (\%) & & & & 0.271 \\
\hline Yes & $49(27.7)$ & $30(23.3)$ & 19 (39.6) & - \\
\hline No & $128(72.3)$ & $99(76.7)$ & $29(60.4)$ & - \\
\hline ASA Physical Status, n (\%) & & & & $0.030^{*}$ \\
\hline ૧ & $4(2.3)$ & $2(1.6)$ & $2(4.2)$ & - \\
\hline Q & $147(83.1)$ & $113(87.6)$ & $34(70.8)$ & - \\
\hline ] & $26(14.7)$ & $14(10.9)$ & $12(25.0)$ & - \\
\hline Type of esophagectomy, n (\%) & & & & 0.501 \\
\hline Mckeown & $164(927)$ & $120(93.0)$ & $44(91.7)$ & - \\
\hline Ivor Lewis & $13(7.3)$ & $9(7.0)$ & $4(8.3)$ & - \\
\hline $\begin{array}{l}\text { Length of operation, min (mean } \pm \\
\text { SD) }\end{array}$ & $363.9 \pm 71.0$ & $358.3 \pm 72.0$ & $379.0 \pm 66.7$ & 0.085 \\
\hline Blood loss, ml (mean \pm SD) & $184.1 \pm 65.0$ & $181.3 \pm 72.2$ & $191.7 \pm 39.0$ & 0.225 \\
\hline
\end{tabular}

Note: Continuous variables are presented as mean \pm standard deviation, and categorical variables are presented as number (\%). $p$-Value were calculated by Chi-square test, Fisher exact test, MannWhitney U or T test.

Abbreviations: $A F R$ albumin-to-fibrinogen ratio, $B M /$ body mass index, $C O P D$ chronic obstructive pulmonary disease, $F V C /$ pre forced votal capacity/predicion, $F E V 1 /$ pre forced expiratory volumn in one second/prediction, ASA American Society of Anesthesiologists, PPC postoperative pulmonary complication, ICU intensive care unit 


\begin{tabular}{|c|c|c|c|c|}
\hline Variables & $\begin{array}{l}\text { All patients [n = } \\
\text { 177] }\end{array}$ & $\begin{array}{l}A F R \geq 11.1[n= \\
129]\end{array}$ & $\begin{array}{l}\mathrm{AFR}<11.1[\mathrm{n}= \\
48]\end{array}$ & $\begin{array}{l}p- \\
\text { Value }\end{array}$ \\
\hline Site of anastomotic, n (\%) & & & & 0.578 \\
\hline Neck & $159(89.8)$ & $117(90.7)$ & $42(87.5)$ & - \\
\hline Chest & $30(5.6)$ & $12(9.3)$ & $6(12.5)$ & - \\
\hline Histology, n (\%) & & & \multicolumn{2}{|l|}{0.742} \\
\hline Squamous cell carcinoma & $154(87.0)$ & $111(86.0)$ & $43(89.6)$ & - \\
\hline Adenocarcinoma & $19(10.7)$ & $14(10.9)$ & $5(10.4)$ & - \\
\hline Others & $4(2.3)$ & $4(3.1)$ & 0 & - \\
\hline Pathological depth of invasion, $\mathrm{n}$ & & & & 0.680 \\
\hline pTo & $19(10.7)$ & $16(12.4)$ & $3(6.3)$ & - \\
\hline $\mathrm{pT} 1$ & $11(6.2)$ & $8(6.2)$ & $3(6.3)$ & - \\
\hline рT2 & $32(18.1)$ & $24(18.6)$ & $8(16.7)$ & - \\
\hline pT3 & $40(22.6)$ & $30(23.3)$ & $10(20.8)$ & - \\
\hline pT4 & $75(42.4)$ & $51(39.5)$ & $24(50.0)$ & - \\
\hline Pathological depth of invasion, $n$ & & & & 0.560 \\
\hline pNO & $118(66.7)$ & $90(69.8)$ & $28(58.3)$ & - \\
\hline pN1 & $32(18.1)$ & $21(16.3)$ & $11(22.9)$ & - \\
\hline pN2 & $20(11.3)$ & $14(10.9)$ & $6(12.5)$ & - \\
\hline pN3 & $7(4.0)$ & $4(3.0)$ & $3(6.3)$ & - \\
\hline Histologic grade, n (\%) & & & & 0.437 \\
\hline G1 & $18(10.2)$ & $12(9.3)$ & $6(12.5)$ & - \\
\hline $\mathrm{G} 1-2$ & $22(12.4)$ & $14(10.9)$ & $8(16.7)$ & - \\
\hline G2 & $125(70.6)$ & $92(71.3)$ & $33(68.8)$ & - \\
\hline
\end{tabular}

G2-3

Nggte: Continuous variables are presented as mean \pm standard deviation, and categorical variables are presented as number (\%). $p$-Value were calculated by Chi-square test, Fisher exact test, MannWhitney U or T test.

Abbreviations: $A F R$ albumin-to-fibrinogen ratio, $B M /$ body mass index, $C O P D$ chronic obstructive pulmonary disease, $F V C /$ pre forced votal capacity/predicion, $F E V 1 /$ pre forced expiratory volumn in one second/prediction, ASA American Society of Anesthesiologists, PPC postoperative pulmonary complication, ICU intensive care unit 


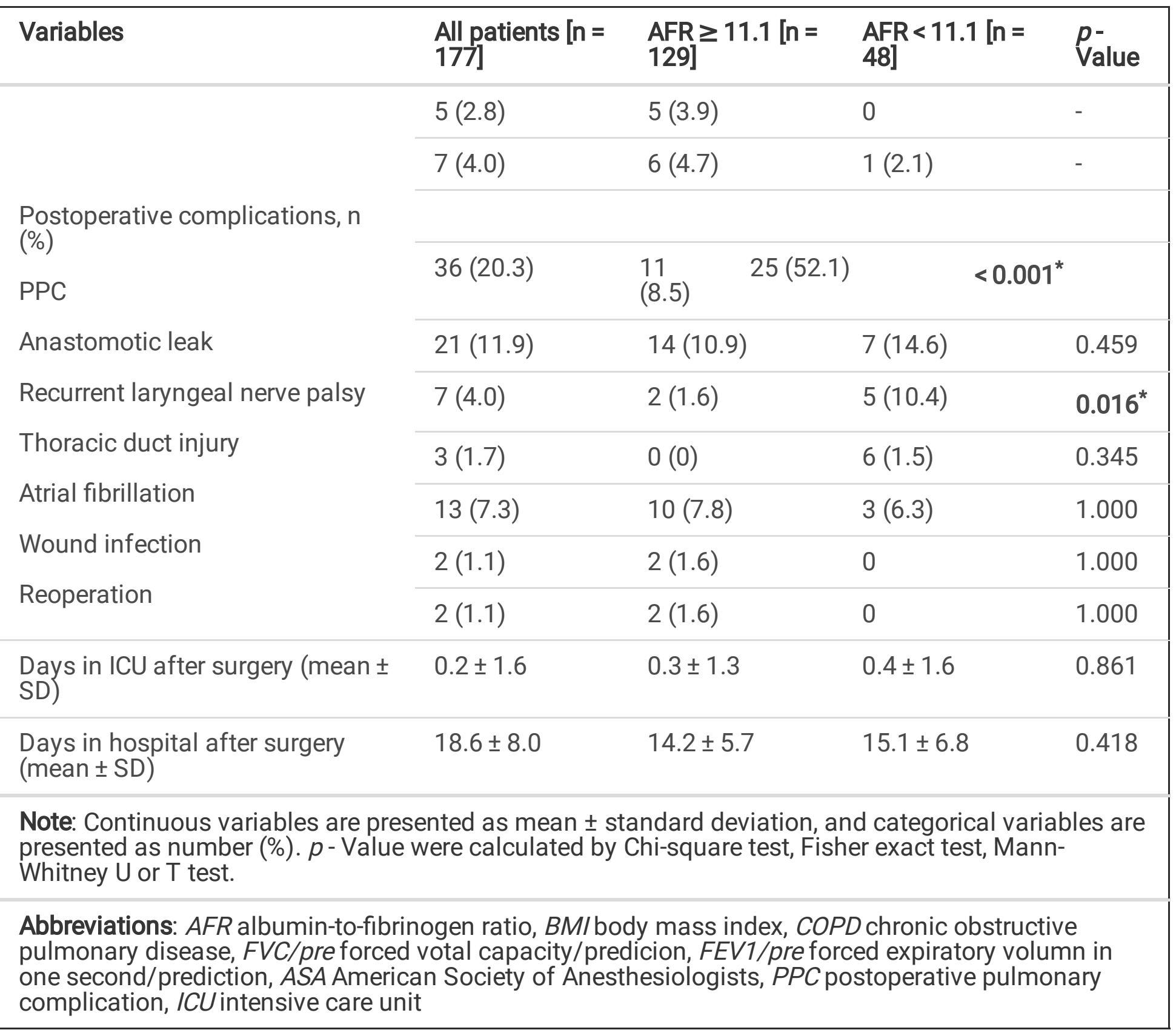

\section{The predictive value of AFR for PPC}

ROC curve analysis was used to assess the predictive value of AFR for PPC. As shown in Fig. 1, preoperative AFR in training group predicted PPC with the area under the curve (AUC) of $0.817,95 \% \mathrm{Cl}$ of $0.771-0.864$, the optimal cut-off value was 11.1 , with a sensitivity of $76.2 \%$, and specificity of $78.7 \%$ ( $p<$ 0.001). The AUC of binary classification of AFR was $0.771,95 \% \mathrm{Cl}$ of $0.721-0.820$, sensitivity $75.4 \%$ and specificity $78.7 \%(p<0.001)$ (Fig. 2).

In validation group, the AFR predicted PPC with the AUC of $0.803,95 \% \mathrm{Cl}$ of $0.711-0.895$, cut-off value 10.8 , sensitivity of $69.4 \%$ and specificity of $85.8 \%$, respectively $(p<0.001)$ (Fig. 3 ). The AUC of binary classification of AFR was $0.766,95 \% \mathrm{Cl}$ of $0.670-0.861$, sensitivity $69.4 \%$ and specificity $83.9 \%(\mathrm{p}<$ 0.001) (Fig. 4). 


\section{Discussion}

With the development of surgical techniques, postoperative complications of esophageal cancer have been significantly reduced ${ }^{4}$. Unfortunately, surgically related complications still occur in more than half of patients with esophageal cancer, with the most common complications being PPCs, which increased the postoperative mortality rate by nearly $10 \%$ and decreased 5 -year overall survival rate by $12 \% 24-26$. Although the incidence of PPCs can be reduced by MIE, persuading patients to quit smoking before surgery, exercising respiratory related muscles before surgery, and relieving postoperative wound pain ${ }^{27}$, there is still lack of a readily available model that can effectively predict the incidence of PPCs. In this

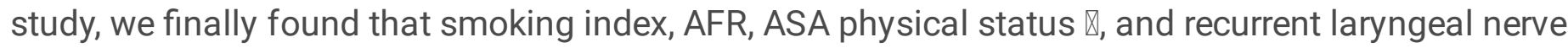
palsy were independent risk factors for PPCs in patients with esophageal cancer using univariate and multivariate logistic analysis. Through two types and two groups of ROC curve analysis, it was found that preoperative AFR could well predict PPCs. The AUC value were 0.817 in the training group and 0.803 in the validation group, which was the highest among the collected laboratory tests data with good sensitivity $(76.2 \%)$, specificity $(78.7 \%)$ and sensitivity $(69.4 \%)$, specificity $(85.8 \%)(p<0.001)$. Binary AFR also demonstrated preferable prediction with AUC 0.771 , sensitivity $(75.4 \%)$, specificity $(78.7 \%)$ and AUC 0.766 , sensitivity $(69.4 \%)$, specificity $(83.9 \%)(p<0.001)$.

The nutritional status of cancer patients is an important factor in determining postoperative prognosis, morbidity and mortality ${ }^{22}$. Although $\mathrm{PNI}$, prognostic nutritional index, has been proven to predict postoperative complications and outcomes in varies cancer patients ${ }^{22,28-30}$, it is not the independent risk factor or the best predictive signature in our study with AUC 0.730. As mentioned above, many studies have been conducted on albumin and AFR as independent influencing factors to predict postoperative complications and prognosis in tumor patients. The results of both groups in this study indicated that lower AFR, which seems means lower albumin or higher fibrinogen, was more prone to PPCs. Increasing albumin to prevent postoperative complications is correlated with previous reports that low serum albumin may lead to a higher risk of worsening disease and poor prognosis in cancer patients ${ }^{13}$. This might be due to malnutrition can weaken the immune system, increase the chance of infection, and albumin may help stabilize the DNA replication and cell growth, regulate the body's reaction, enhance immunity, prevention of malignant disease ${ }^{18}$. Fibrinogen, a fibrin-based soluble clotting substrate that plays a central role in hemostasis and thrombosis, is associated with cardiovascular event risk and prethrombosis status in experimental models ${ }^{31}$, and is also widely reported as an acute phase protein involved in inflammatory response. Fibrinogen synthesis is regulated by a number of inflammatory cytokines, including interleukin-1 (IL-1) and IL- $6^{32}$. Thus, elevated FIB within a range may indicate a higher likelihood of thrombosis and attack by inflammatory factors. Theoretically, it may hamper the patient's recovery after surgery. As for AFR, a new indicator, represents the combined effect of the two blood factors mentioned, enhancing sensitivity to assess inflammation and nutritional status. In various models of acute ST-segment elevation myocardial infarction (STEMI) ${ }^{33}$, non small cell lung cancer $(\mathrm{NSCLC})^{34}$, soft tissue sarcoma ${ }^{35}$, and esophageal cancer ${ }^{18}$, the combination of albumin and fibrinogen has been reported to be superior to albumin and fibrinogen alone, and has been widely recommended as 
a prognostic factor. It should be noted that in a report on the prediction of prognosis of esophageal cancer by $\mathrm{AFR}^{18}$, postoperative complications of patients were not included in the study, so it was not possible to assess whether the prognosis of patients after esophageal cancer was caused by the occurrence of postoperative complications. However, it remains to be further explored whether AFR or PPCs has a stronger correlation with prognosis. To our knowledge, the present study is the first to demonstrate the association between AFR and PPCs after MIE.

Plenty of studies have demonstrated that current smoking status is associated with PPCs ${ }^{36}$, and smoking cessation decrease the incidence of PPCs ${ }^{27,37}$. But none of these studies seemed to include smoking index that reflects the degree of smoking rather than the status of smoking in some aspect. When including both smoking status and smoking index in the logistic regression analysis, we found that only smoking index (OR 1.001, 95\% Cl 1.000-1.002, $p=0.024$ ) was a significant risk factor and was also significantly different in validation group which might mean that the amount of smoking, rather than smoking status, is a better predictor of PPCs, although it is needed validation among large sample and multiple centers.

Similar to previous studies ${ }^{38-41}$, our work confirmed that ASA was an independent risk factor for PPCs, while only grade $\otimes$ was significant taking grade $\nabla$ as reference. ASA system was to assess the general health and comorbidities of patients before surgery ${ }^{39}$. Our study provides evidence for the foregoing and future related research.

The recurrent laryngeal nerve plays an important role in spinal cord coordination. RLN originates from the vagus nerve and provides ipsilateral motor innervation to the internal laryngeal muscles except the cricothyroid. These muscles play an important role in speech, swallowing and breathing ${ }^{27}$. As reported before, the incidence was from $0-29.3 \% 42$, and Ivor Lewis could decrease the incidence to $0.9 \% 43$. However, the occurrence of recurrent laryngeal nerve palsy showed a strong correlation with postoperative pneumonia ${ }^{44}$. In this study, the incidence was $3.2 \%$ in training group and $4 \%$ in validation group. Its association with PPCs was verified by both sets of data $(p<0.05)$.

However, there are still several deficiencies in our study. First of all, this study is a retrospective, singlecenter study, which is likely to cause selective bias, and it needs to be verified by prospective, multicenters, large-sample trials in the future, which may explain why age, body mass index (BMI) and FEV1/pre ${ }^{5,17,22}$ were not independent risk factors as reported before. In addition, it is not clear whether preoperative intervention to change AFR level can reduce the incidence of PPCs. Finally, the mechanism that how AFR can predict postoperative pneumonia in patients with esophageal cancer is still unclear, and the molecular mechanism may be found through relevant basic experiments.

\section{Conclusions}

First of all, this study found that preoperative AFR can effectively predict the occurrence of PPCs in patients with esophageal cancer as an independent influence factor. Secondly, our study confirmed that 
ASA, smoking index and recurrent laryngeal nerve palsy are independent risk factors for PPC

\section{Abbreviations}

MIE: minimally invasive esophagectomy; RCT: randomized controlled trial; PPC: postoperative pulmonary complication; CT: computed tomography; AFR: Albumin-to-fibrinogen ratio; ICU: intense care unit; rUPS: the revised Uniform Pneumonia Score; PNI: prognostic nutritional index; AGR: albumin-to-globulin ratio; ASA: American Society of Anesthesiologists; NLR: neutrophils-to-lymphocyte ratio; PLR: platelet-tolymphocyte; MLR: monocyte-to-lymphocyte ratio; ROC: Receiver operating characteristic; SD: standard deviation BMI: Body mass index; Cl: Confidence interval; OR: Odds ratio; STEMI: ST-segment elevation myocardial infarction; NSCLC: nonsmall cell lung cancer.

\section{Declarations}

\section{Ethics approval and consent to participate}

This retrospective study was approved by the the Ethics Committee of Scientific Research and Clinical Trial of The First Affiliated Hospital of Zhengzhou University. Written informed consent was obtained from all eligible patients. All of the procedures were performed in accordance with the Declaration of Helsinki and relevant policies in China.

\section{Consent for publication}

Not applicable

\section{Availability of data and materials}

All data generated or analysed during this study are included in this published article.

\section{Competing interests}

The authors declare that they have no competing interests

\section{Funding}

This study received no external funding

\section{Authors' contributions}

Conceptualization, Methodology, Software, Validation, Formal Analysis, Investigation, Data Writing Original Draft Preparation, Zhang Peng

Writing - Review \& Editing, Visualization, Supervision, Project Administration, Zhao Song

\section{Acknowledgements}




\section{References}

1. Wong MCS, Hamilton W, Whiteman DC, et al. Global Incidence and mortality of oesophageal cancer and their correlation with socioeconomic indicators temporal patterns and trends in 41 countries. Sci Rep 2018; 8(1): 4522.

2. Bray F, Ferlay J, Soerjomataram I, Siegel RL, Torre LA, Jemal A. Global cancer statistics 2018 : GLOBOCAN estimates of incidence and mortality worldwide for 36 cancers in 185 countries. $C A$ Cancer J Clin 2018; 68(6): 394-424.

3. Yang H, Liu H, Chen Y, et al. Neoadjuvant Chemoradiotherapy Followed by Surgery Versus Surgery Alone for Locally Advanced Squamous Cell Carcinoma of the Esophagus (NEOCRTEC5010): A Phase III Multicenter, Randomized, Open-Label Clinical Trial. J Clin Oncol 2018; 36(27): 2796-803.

4. Biere SS, van Berge Henegouwen MI, Maas KW, et al. Minimally invasive versus open oesophagectomy for patients with oesophageal cancer: a multicentre, open-label, randomised controlled trial. Lancet (London, England) 2012; 379(9829): 1887-92.

5. Kanda M, Koike M, Tanaka C, et al. Risk Prediction of Postoperative Pneumonia After Subtotal Esophagectomy Based on Preoperative Serum Cholinesterase Concentrations. Ann Surg Oncol 2019; 26(11): 3718-26.

6. Kataoka K, Takeuchi H, Mizusawa J, et al. Prognostic Impact of Postoperative Morbidity After Esophagectomy for Esophageal Cancer: Exploratory Analysis of JCOG9907. Ann Surg 2017; 265(6): $1152-7$.

7. Nozoe T, Kimura Y, Ishida M, Saeki H, Korenaga D, Sugimachi K. Correlation of pre-operative nutritional condition with post-operative complications in surgical treatment for oesophageal carcinoma. Eur J Surg Oncol 2002; 28(4): 396-400.

8. Hashimoto S, Tominaga T, Nonaka T, et al. The C-reactive protein to albumin ratio predicts postoperative complications in oldest-old patients with colorectal cancer. International journal of colorectal disease 2020; 35(3): 423-31.

9. Kijima T, Arigami T, Uchikado Y, et al. Combined fibrinogen and neutrophil-lymphocyte ratio as a prognostic marker of advanced esophageal squamous cell carcinoma. Cancer Sci 2017; 108(2): 193-9.

10. Kurita D, Oguma J, Ishiyama K, Hirano Y, Kanamori J, Daiko H. Handgrip Strength Predicts Postoperative Pneumonia After Thoracoscopic-Laparoscopic Esophagectomy for Patients with Esophageal Cancer. Ann Surg Oncol 2020; 27(9): 3173-81.

11. Yasukawa K, Shimizu A, Motoyama H, et al. Preoperative C-reactive protein-to-albumin ratio predicts long-term outcomes in extrahepatic cholangiocarcinoma patients. J Surg Oncol 2020; 122(6): 1094105. 
12. Goh SL, De Silva RP, Dhital K, Gett RM. Is low serum albumin associated with postoperative complications in patients undergoing oesophagectomy for oesophageal malignancies? Interactive cardiovascular and thoracic surgery 2015; 20(1): 107-13.

13. Lin MY, Liu WY, Tolan AM, Aboulian A, Petrie BA, Stabile BE. Preoperative serum albumin but not prealbumin is an excellent predictor of postoperative complications and mortality in patients with gastrointestinal cancer. The American surgeon 2011; 77(10): 1286-9.

14. Quhal F, Pradere B, Sari Motlagh R, et al. Prognostic value of preoperative albumin to globulin ratio in patients treated with salvage radical prostatectomy for radiation recurrent prostate cancer. Minerva urologica e nefrologica = The Italian journal of urology and nephrology 2020.

15. Baik H, Bae KB. Low albumin level and longer interval to closure increase the early complications after ileostomy closure. Asian journal of surgery 2021; 44(1): 352-7.

16. Guan $X$, Gong $M$, Wang $X$, et al. Low preoperative fibrinogen level is risk factor for neurological complications in acute aortic dissection. Medicine 2018; 97(21): e10830.

17. You X, Zhou Q, Song J, Gan L, Chen J, Shen H. Preoperative albumin-to-fibrinogen ratio predicts severe postoperative complications in elderly gastric cancer subjects after radical laparoscopic gastrectomy. BMC Cancer 2019; 19(1): 931.

18. Tan Z, Zhang M, Han Q, et al. A novel blood tool of cancer prognosis in esophageal squamous cell carcinoma: the Fibrinogen/Albumin Ratio. J Cancer 2017; 8(6): 1025-9.

19. Song YN, Qi Y, Zhang CY, et al. A new technology for reducing anastomotic fistula in the neck after esophageal cancer surgery. J Thorac Dis 2019; 11(7): 3084-92.

20. Canet J, Gallart L, Gomar C, et al. Prediction of postoperative pulmonary complications in a population-based surgical cohort. Anesthesiology 2010; 113(6): 1338-50.

21. Weijs TJ, Seesing MF, van Rossum PS, et al. Internal and External Validation of a multivariable Model to Define Hospital-Acquired Pneumonia After Esophagectomy. J Gastrointest Surg 2016; 20(4): 6807.

22. Yu J, Hong B, Park JY, Hwang JH, Kim YK. Impact of Prognostic Nutritional Index on Postoperative Pulmonary Complications in Radical Cystectomy: A Propensity Score-Matched Analysis. Ann Surg Oncol 2021; 28(3): 1859-69.

23. Onodera T, Goseki N, Kosaki G. [Prognostic nutritional index in gastrointestinal surgery of malnourished cancer patients]. Nihon Geka Gakkai zasshi 1984; 85(9): 1001-5.

24. Low DE, Kuppusamy MK, Alderson D, et al. Benchmarking Complications Associated with Esophagectomy. Ann Surg 2019; 269(2): 291-8.

25. Goense L, Meziani J, Ruurda JP, van Hillegersberg R. Impact of postoperative complications on outcomes after oesophagectomy for cancer. Br J Surg 2019; 106(1): 111-9.

26. Booka E, Takeuchi H, Nishi T, et al. The Impact of Postoperative Complications on Survivals After Esophagectomy for Esophageal Cancer. Medicine 2015; 94(33): e1369. 
27. Chevallay M, Jung M, Chon SH, Takeda FR, Akiyama J, Mönig S. Esophageal cancer surgery: review of complications and their management. Ann N Y Acad Sci 2020; 1482(1): 146-62.

28. Takagi K, Domagala P, Polak WG, Buettner S, ljzermans JNM. The Controlling Nutritional Status Score and Postoperative Complication Risk in Gastrointestinal and Hepatopancreatobiliary Surgical Oncology: A Systematic Review and Meta-Analysis. Annals of nutrition \& metabolism 2019; 74(4): $303-12$.

29. Saito H, Kono Y, Murakami Y, et al. Influence of prognostic nutritional index and tumor markers on survival in gastric cancer surgery patients. Langenbeck's archives of surgery 2017; 402(3): 501-7.

30. Okada S, Shimada J, Teramukai S, et al. Risk Stratification According to the Prognostic Nutritional Index for Predicting Postoperative Complications After Lung Cancer Surgery. Ann Surg Oncol 2018; 25(5): 1254-61.

31. Weisel JW, Litvinov RI. Fibrin Formation, Structure and Properties. Sub-cellular biochemistry 2017; 82 : 405-56.

32. Tang MJ, Ding SB, Hu WY. Fibrinogen and Albumin Score Changes during Preoperative Treatment Can Predict Prognosis in Patients with Locally Advanced Rectal Cancer. Gastroenterology research and practice 2019; 2019: 3514586.

33. Zhao Y, Yang J, Ji Y, et al. Usefulness of fibrinogen-to-albumin ratio to predict no-reflow and shortterm prognosis in patients with ST-segment elevation myocardial infarction undergoing primary percutaneous coronary intervention. Heart and vessels 2019; 34(10): 1600-7.

34. Ying J, Zhou D, Gu T, Huang J, Liu H. Pretreatment albumin/fibrinogen ratio as a promising predictor for the survival of advanced non small-cell lung cancer patients undergoing first-line platinum-based chemotherapy. BMC Cancer 2019; 19(1): 288.

35. Liang $Y$, Wang W, Que $Y$, et al. Prognostic value of the fibrinogen/albumin ratio (FAR) in patients with operable soft tissue sarcoma. BMC Cancer 2018; 18(1): 942.

36. Grønkjær M, Eliasen M, Skov-Ettrup LS, et al. Preoperative smoking status and postoperative complications: a systematic review and meta-analysis. Ann Surg 2014; 259(1): 52-71.

37. Mills E, Eyawo O, Lockhart I, Kelly S, Wu P, Ebbert JO. Smoking cessation reduces postoperative complications: a systematic review and meta-analysis. The American journal of medicine 2011; 124(2): 144 - 54.e8.

38. Schoenfeld AJ, Carey PA, Cleveland AW, 3rd, Bader JO, Bono CM. Patient factors, comorbidities, and surgical characteristics that increase mortality and complication risk after spinal arthrodesis: a prognostic study based on 5,887 patients. The spine journal: official journal of the North American Spine Society 2013; 13(10): 1171-9.

39. Kay HF, Sathiyakumar V, Yoneda ZT, et al. The effects of American Society of Anesthesiologists physical status on length of stay and inpatient cost in the surgical treatment of isolated orthopaedic fractures. Journal of orthopaedic trauma 2014; 28(7): e153-9.

40. Davenport DL, Bowe EA, Henderson WG, Khuri SF, Mentzer RM, Jr. National Surgical Quality Improvement Program (NSQIP) risk factors can be used to validate American Society of 
Anesthesiologists Physical Status Classification (ASA PS) levels. Ann Surg 2006; 243(5): 636 - 41; discussion $41-4$.

41. Portier K, Ida KK. The ASA Physical Status Classification: What Is the Evidence for Recommending Its Use in Veterinary Anesthesia?-A Systematic Review. Frontiers in veterinary science 2018; 5: 204.

42. Pertl L, Zacherl J, Mancusi G, et al. High risk of unilateral recurrent laryngeal nerve paralysis after esophagectomy using cervical anastomosis. European archives of oto-rhino-laryngology: official journal of the European Federation of Oto-Rhino-Laryngological Societies (EUFOS) : affiliated with the German Society for Oto-Rhino-Laryngology - Head and Neck Surgery 2011; 268(11): 1605-10.

43. Visbal AL, Allen MS, Miller DL, Deschamps C, Trastek VF, Pairolero PC. Ivor Lewis esophagogastrectomy for esophageal cancer. Ann Thorac Surg 2001; 71(6): 1803-8.

44. Oshikiri T, Takiguchi $\mathrm{G}$, Hasegawa $\mathrm{H}$, et al. Postoperative recurrent laryngeal nerve palsy is associated with pneumonia in minimally invasive esophagectomy for esophageal cancer. Surg Endosc 2021; 35(2): 837-44.

\section{Figures}

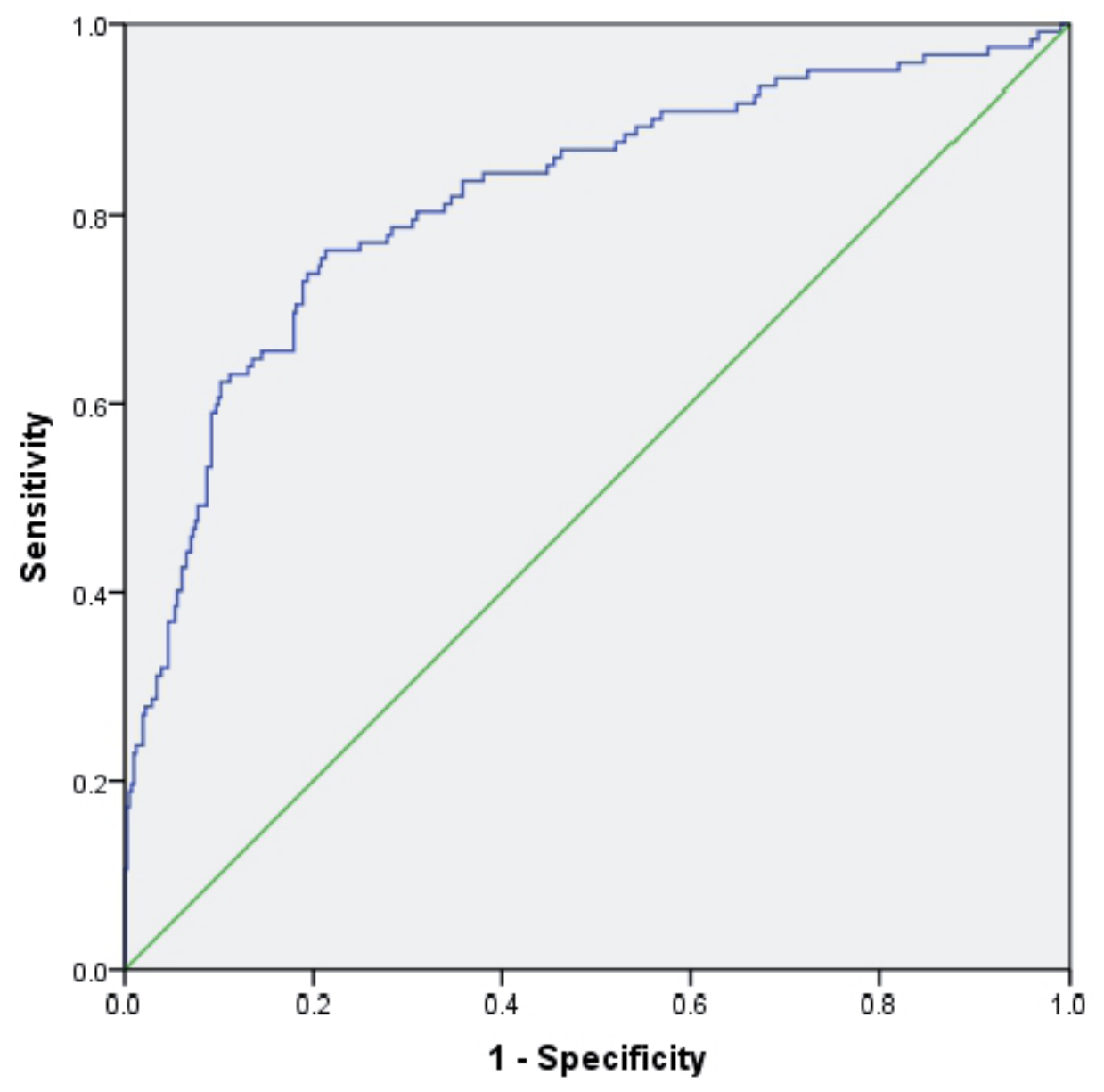

Figure 1 
ROC curve analysis of AFR for PPC in training group Notes: Predictive value of AFR for PPC in training group by ROC curve analysis. The result indicated preoperative AFR as a potential predictive factor with an AUC of $0.817,95 \% \mathrm{Cl}$ of $0.771-0.864$, cut-off value of 11.1 , sensitivity $76.2 \%$ and specificity $78.7 \%$, respectively $(p<0.001)$. Abbreviations: ROC receiver operating characteristic, AFR, albumin-to-fibrinogen ratio, $\mathrm{PPC}$ postoperative pulmonary complication, AUC the area under the curve, Cl confidence interval

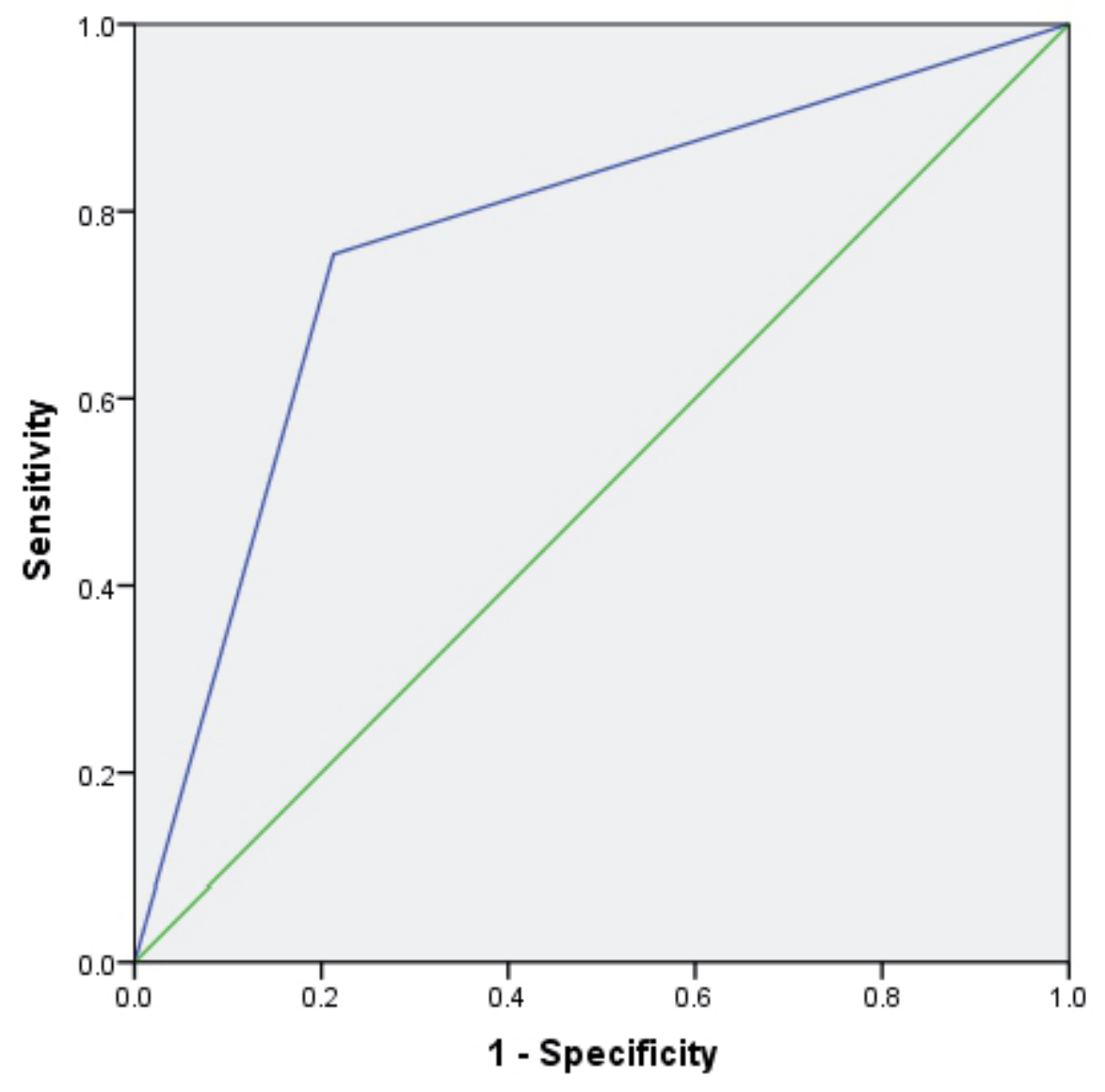

\section{Figure 2}

ROC curve analysis of AFR based on cut-off value for PPC in training group Notes: Predictive value of AFR based on cut-off value by ROC curve analysis. AUC $0.771,95 \% \mathrm{Cl} 0.721-0.820$, sensitivity $75.4 \%$ and specificity $78.7 \%$, respectively $(p<0.001)$. Abbreviations: ROC receiver operating characteristic, AFR albumin-to-fibrinogen ratio,PPC postoperative pulmonary complication, AUC the area under the curve, $\mathrm{Cl}$ confidence interval 




Figure 3

ROC curve analysis of AFR for PPC in validated group Notes: The results indicated preoperative AFR as a potential predictive factor with AUC $0.803,95 \% \mathrm{Cl} 0.711-0.895$, cut-off value 10.8 , sensitivity of $69.4 \%$ and specificity of $85.8 \%$, respectively $(p<0.001)$. Abbreviations: ROC receiver operating characteristic, AFR albumin-to-fibrinogen ratio, PPC postoperative pulmonary complication, AUC the area under the curve, $\mathrm{Cl}$ confidence interval 


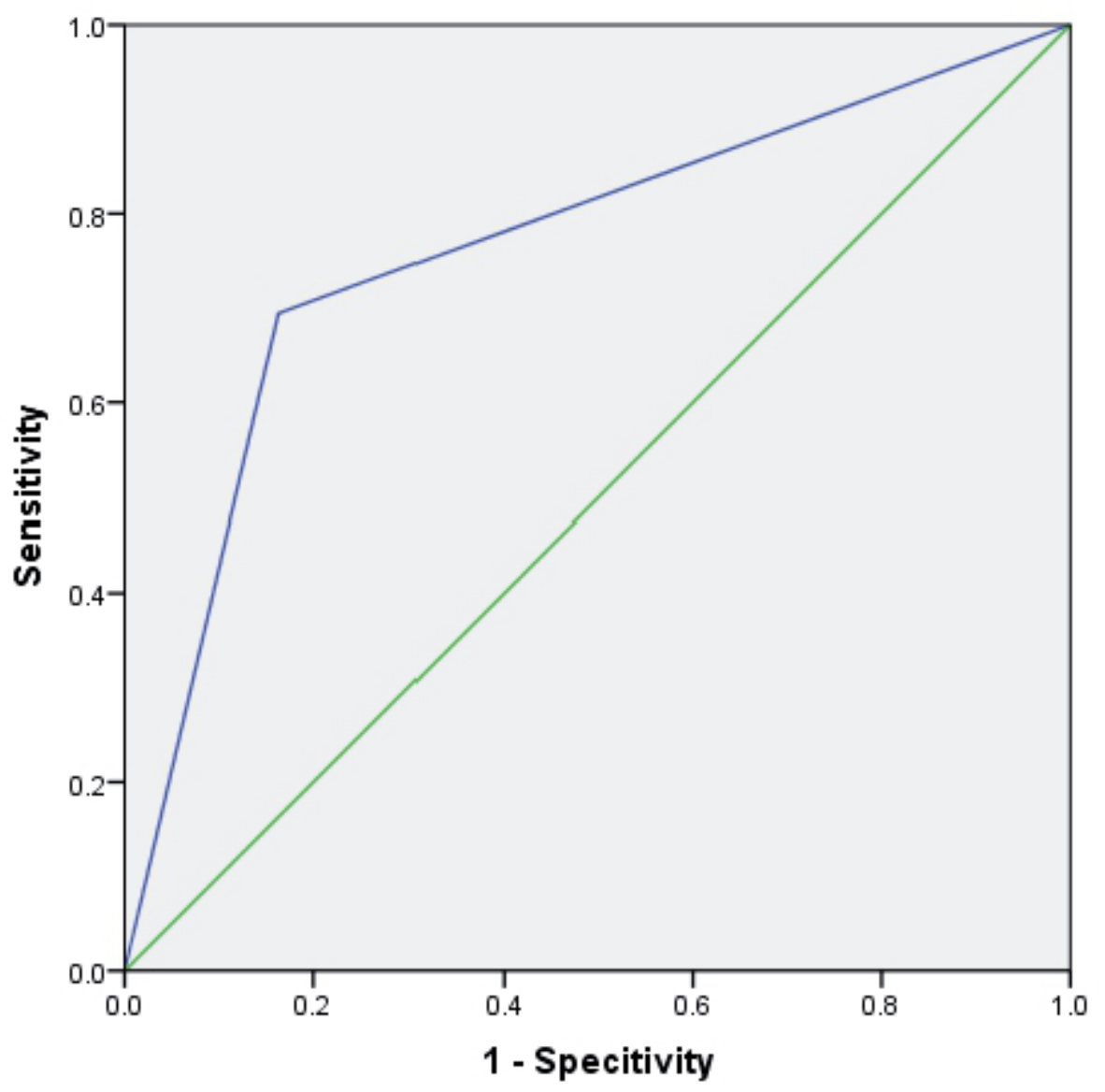

\section{Figure 4}

ROC curve analysis of AFR based on cut-off value for PPC in validated group Notes: The results indicated AUC $0.766,95 \% \mathrm{Cl} 0.670-0.861$, sensitivity $69.4 \%$ and specificity $83.9 \%$ respectively $(p<0.001)$. Abbreviations: ROC receiver operating characteristic, AFR albumin-to-fibrinogen ratio, PPC postoperative pulmonary complication, AUC the area under the curve, $\mathrm{Cl}$ confidence interval 